\title{
Knockdown of Pin1 leads to reduced angiogenic potential and tumorigenicity in glioblastoma cells
}

\author{
KUTAY DENIZ ATABAY ${ }^{1-3}$, MEHMET TAHA YILDIZ ${ }^{1,4^{*}}$, TIMUCIN AVSAR $^{1,2^{*}}$, \\ ARZU KARABAY ${ }^{2}$ and TÜRKER KILIÇ ${ }^{1,5}$ \\ ${ }^{1}$ Prof. Dr. Peter Black Laboratory of Molecular Neurosurgery, Institute of Neurological Sciences, Marmara University; \\ ${ }^{2}$ Department of Molecular Biology and Genetics, Faculty of Science and Letters, Istanbul Technical University; \\ ${ }^{4}$ Department of Biology, Faculty of Arts and Science, Fatih University; ${ }^{5}$ Department of Neurosurgery, \\ Faculty of Medicine, Bahcesehir University, Istanbul, Turkey
}

Received June 30, 2014; Accepted March 30, 2015

DOI: $10.3892 / \mathrm{ol} .2015 .3512$

\begin{abstract}
Glioblastoma is the most common and most aggressive type of primary brain tumor. Current approaches in the treatment of glioblastoma are not effective enough to increase patient survival or prevent recurrence following surgery. Consequently, the search for potential drug targets is ongoing. Peptidyl-prolyl cis/trans isomerase NIMA-interacting 1 (Pin 1 ), an isomerase that is overexpressed in various tumors, has become an attractive molecule in cancer research. Pin 1 has been reported to regulate proteins involved in essential cellular pathways that mediate cell proliferation, cell cycle progression, differentiation and apoptosis, by altering their stability and function. The results of the present study revealed that knockdown of Pin1 in glioblastoma cells using RNA interference or the selective Pin1 inhibitor, juglone, suppressed the tumorigenic features by reducing cell growth, migration and angiogenic potential. Furthermore, knockdown of Pin1 decreased the levels of vascular endothelial growth factor and matrix metallopeptidase 9, and also triggered apoptosis. Due to the fundamental roles of Pin1 in promoting tumorigenesis, Pin1 inhibitory molecules, including juglone, or alternative synthetic derivatives hold potential for the development of clinical countermeasures against glioblastoma.
\end{abstract}

Correspondence to: Professor Türker Kiliç, Department of Neurosurgery, Faculty of Medicine, Bahcesehir University, Curagan Caddesi, Osmanpasa Mektebi Sokak 4-6 Besiktas, Istanbul 34353, Turkey

E-mail: turker.kilic@tnn.net

Present address: ${ }^{3}$ Department of Brain and Cognitive Sciences, Massachusetts Institute of Technology, Cambridge, MA 02139, USA

*Contributed equally

Key words: glioblastoma, tumorigenesis, peptidyl-prolyl cis/trans isomerase NIMA-interacting 1, juglone, vascular endothelial growth factor

\section{Introduction}

Glioblastoma is the most common and most aggressive type of primary brain tumor, accounting for nearly $65 \%$ of all primary intracranial tumors and conferring a median survival time of $\sim 14$ months (1). The molecular mechanisms underlying the pathology of glioblastoma, which is not completely curable by means of chemotherapy, radiotherapy and surgery, are yet to be fully elucidated. Therefore, the search for candidate molecules to be employed as countermeasures against glioblastoma remains essential. In addition, it is equally important to identify potential target molecules responsible for the pathogenesis of glioblastoma in order to develop novel clinical treatment modalities.

Peptidyl-prolyl cis/trans isomerase NIMA-interacting 1 (Pin1) is a member of the parvulin family; the peptidyl-prolyl cis/trans isomerase (PPIase) group of proteins (2). Previous data has identified that Pin1 is broadly overexpressed in various types of tumors, including gliomas (3). Members of the Parvulin family have a variety of functions, including modulating the assembly, folding, activation/inactivation and transport of essential proteins to their intracellular targets (4). In addition, the proteins regulate intracellular signaling, transcription, cell cycle progression and apoptosis by altering the function and/or stability of target proteins (5). Pin1 induces conformational changes in its target phospho-proteins by binding and isomerizing the peptidyl-prolyl bond in phosphorylated Ser/Thr-Pro motifs. Consequently, it has roles in a wide range of cellular activities, since Ser/Thr-Pro motifs are also specific phosphorylation sites on a number of protein kinases.

Pin1 expression has been identified to be positively correlated with the expression of vascular endothelial growth factor (VEGF), a key molecule involved in angiogenesis. Pin1 is also known to indirectly regulate VEGF expression through the isomerization of the hypoxia-inducible factor 1 (HIF-1) and activating protein-1 (AP1) transcription factors (6). Furthermore, the overexpression of Pin1 in breast cancer cell lines has been demonstrated to lead to an upregulation in VEGF and a consequent promotion of angiogenesis during cancer progression (6). A previous study also revealed that 
the stable inhibition of Pin1 using retrovirus-mediated small interfering RNA (siRNA) suppressed cell growth, migration ability and angiogenesis in prostate cancer cells (7). Pin1 dependency has been identified to be a specific feature of cancer cells, which may be important with respect to cancer cell selective treatments that utilize Pin1-targeted drugs (7). Juglone (also known as 5-hydroxy-1,4-naphthoquinone), which blocks the function of Pin1, has emerged as an efficient candidate inhibitor molecule. Juglone has been reported to selectively inhibit Pin1 by irreversibly modifying its sulfhydryl groups, whilst not affecting the function of other PPIase family members (8). Juglone has also been used in other studies, due to its selective inhibitory function of Pin1 $(9,10)$.

The aim of the present study was investigate the potential role of Pin1 in glioblastoma pathology. The expression levels of VEGF and matrix metalloproteinase 9 (MMP9) were investigated to determine the effects of Pin1 inhibition on the cell growth, migration and angiogenic potential of glioblastoma cells. In addition, the effect of Pin1 inhibition on the apoptotic response of glioblastoma cells was also investigated. We hypothesize that Pin1 may exhibit a crucial role in the pathogenesis of glioblastoma and thus may present a novel potential molecular target for the treatment of glioblastoma. Furthermore, Pin1 inhibitory molecules, including Juglone or alternative synthetic derivatives, may present promising therapeutic agents for the treatment of glioblastoma and other tumor types that exhibit similar pathological features.

\section{Materials and methods}

Cell cultures. U87-MG glioblastoma cells bearing epithelial morphology (American Type Culture Collection, Manassas, VA, USA) were used for all experiments, as the primary aim of the present study was to elucidate the tumorigenic properties of glioblastoma cells. The cells were plated into high-glucose Dulbecco's modified Eagle's medium (DMEM) supplemented with $2 \mathrm{mM}$ non-essential amino acids and $10 \%$ fetal bovine serum (FBS) and maintained at $37^{\circ} \mathrm{C}$ in $5 \% \mathrm{CO}_{2}$.

RNA interference (RNAi) and juglone treatment. Pin1 siRNA and non-targeting siRNA (GE Dharmacon, Lafayette, CO, USA) fragments were used for knockdown. The transfections were performed using DharmaFect Transfection Reagent-1 (GE Dharmacon) according to the manufacturer's instructions. Separately, for juglone treatment, $0.5 \mathrm{mM}$ juglone (EMD Millipore, Billerica, MA, USA) main stock solution was prepared by first dissolving juglone in DMSO. The solution was then brought up to the final volume with $\mathrm{dH}_{2} \mathrm{O}$.

MTT assay. The human glioblastoma U87-MG cells were cultured in DMEM supplemented with $2 \mathrm{mM}$ non-essential amino acids, $10 \%$ FBS and $1 \%$ penicillin/streptomycin. The cells were treated with juglone, Pin1 siRNA or non-targeting siRNA. Cell proliferation was measured at different time-points using the Vybrant-MTT Cell Proliferation Assay kit (Invitrogen Life Technologies, Carlsbad, CA, USA). All experiments were performed three times in triplicate. A one-way analysis of variance was performed in order to determine the statistical significance of the differences between groups.
Microscopy and apoptosis analysis. The acridine orange/ethidium bromide vital staining technique was used to determine the ratio of living to apoptotic cells, as previously described (11). The cells were incubated for 3 days with Pin1 siRNA or non-targeting siRNA in order to observe knockdown at the protein level.

Wound-healing assay. The U87-MG glioblastoma cells were plated in 12-well plates and cultured for $48 \mathrm{~h}$. A wound was made using the tip of a pipette, as previously described (12). After $36 \mathrm{~h}$, images of the wounds were captured by light microscopy (CH30; Olympus Corporation, Tokyo, Japan).

Western blotting. Protein isolation was performed using the Mammalian Cell Extraction kit (BioVision, Inc., Milpitas, CA, USA). The protein lysates were subjected to $12 \%$ SDS-polyacrylamide gel electrophoresis and transferred to a nitrocellulose membrane. Next, the membrane was blocked with $2.5 \%$ skimmed milk powder and $1 \%$ bovine serum albumin, and then probed with the following primary antibodies at a 1:500 dilution: Pin1 mouse polyclonal immunoglobulin G (IgG; Santa Cruz Biotechnology, Inc., Dallas, TX, USA), $\beta$-actin mouse polyclonal IgG (Santa Cruz Biotechnology, Inc.) and MMP9 rabbit polyclonal IgG (Abcam, Cambridge, UK), in blocking solution. The horseradish peroxidase (HRP)-conjugated secondary antibodies (Promega Corporation, Madison, WI, USA) were used at 1:5,000 dilutions in blocking solution. Amersham ECL Plus Western Blotting Detection Reagents (GE Healthcare Life Sciences, Chalfont, UK) were used to obtain a signal following the HRP/luminogen reaction.

\section{Results}

Pinl inhibition decreases glioblastoma cell growth and activates apoptosis in vitro. The overexpression and inhibition of Pin1 has previously been associated with increased VEGF (6) and decreased MMP9 activity (13), respectively. Pin1 overexpression is also associated with the promotion of certain tumorigenic features that are shared by various tumor types (3). The present study also observed high levels of Pin1, VEGF and MMP9 in the glioblastoma cells (Fig. 1). Pin1 has been associated with essential cellular events that underlie tumorigenicity. Therefore, it was hypothesized that the knockdown of Pin1 in glioblastoma cells would inhibit certain pathological features that are important for tumor cell survival. The effects of Pin1 inhibition on glioblastoma cells were investigated (Fig. 1A). The results were consistent with those of a previous study (7), which identified reduced growth rates following Pin1-knockdown in prostate cancer cell lines. In the present study, there was a slight increase in cell growth on the final day of treatment with $5 \mu \mathrm{M}$ juglone, but not with any other concentration. An increase in the MMP9 protein level was also observed at the same concentration of juglone. Speculatively, inhibition at this specific concentration may trigger a survival response that results in an increase in growth. However, the wound-healing assay revealed that the overall number of cells was lower following Pin1 inhibition, which is consistent with the reduction trend observed in the growth assay. 
A

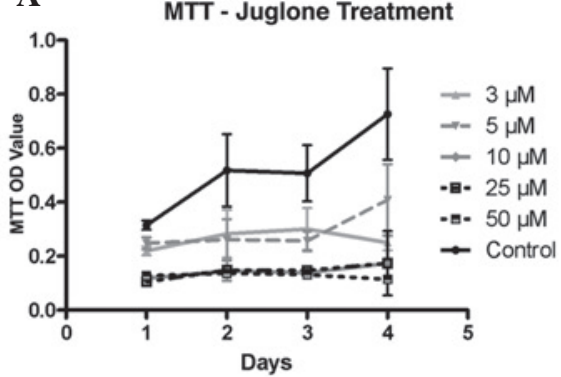

C
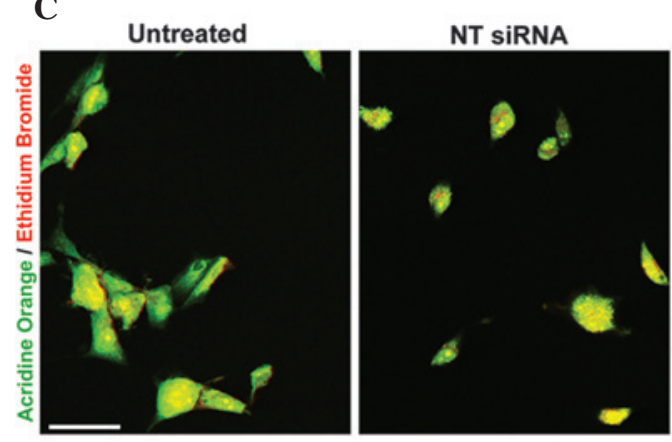

MTT - Pin1 RNAi

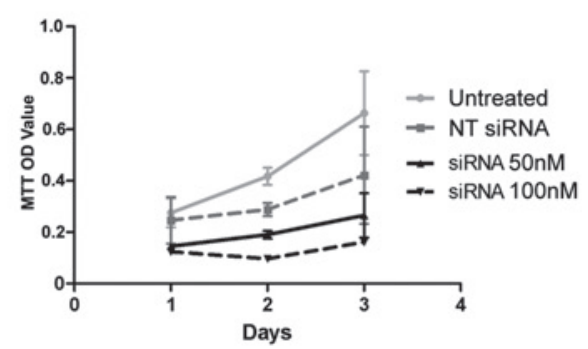

Pin1 siRnA

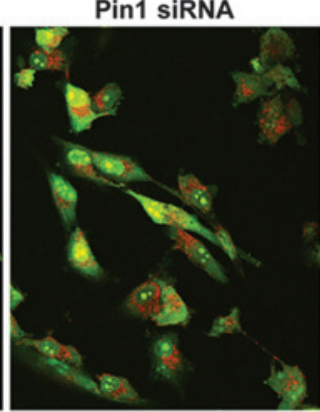

B

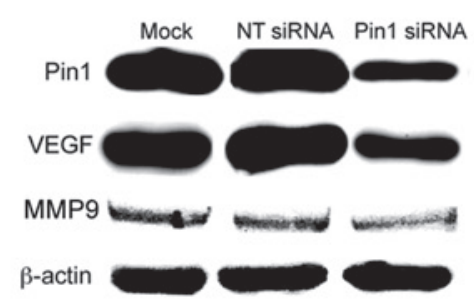

E

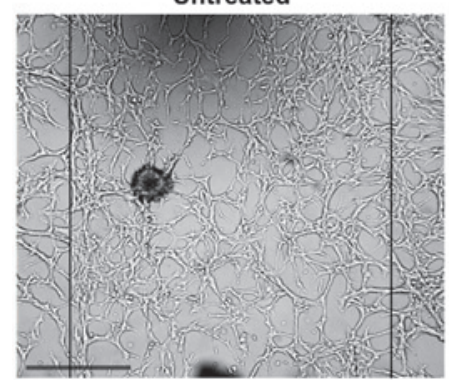

Non-targeting siRNA

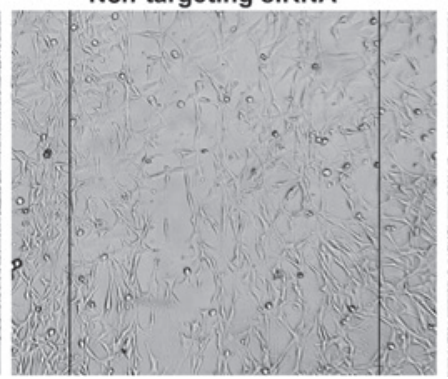

Pin1 Knock Down Initiates Apoptosis

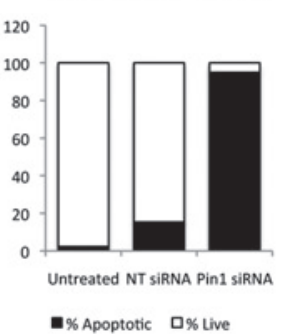

D

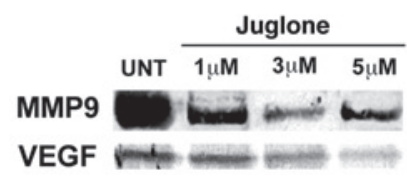

Juglone $5 \mu \mathrm{M}$
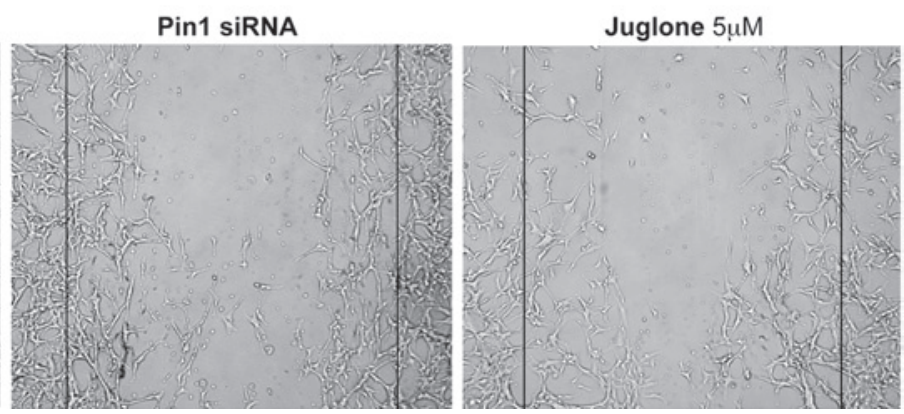

Figure 1. (A) Graphs revealing the reduction in proliferation caused by Pin1 siRNA and juglone in glioblastoma cells in vitro. The difference between juglone-treated and -untreated groups and siRNA-treated versus non-targeting siRNA or untreated groups was significant $(\mathrm{P}<0.05)$. (B) Immunoblotting revealing the reduction in the levels of Pin1, VEGF and MMP9 by Pin1 siRNA. (C) Pin1 siRNA transfection activated apoptosis in the glioblastoma cells, whereas the non-targeting siRNA-treated and control cells were healthy. Acridine orange/ethidium bromide vital staining reveals apoptotic cells with red and orange dots in the perinuclear region, healthy cells in green and necrotic cells in red. In total, 95\% of the cells that were counted in the Pin1 siRNA group were apoptotic ( $\mathrm{n}=115$ ) (scale bar, $200 \mu \mathrm{m}$ ). (D) A significant reduction in VEGF and MMP9 levels observed following juglone treatment is shown. (E) Wound healing assay revealing that Pin1 siRNA and juglone were effective at disrupting the migration ability of glioblastoma cells (scale bar, $50 \mu \mathrm{m}$ ). Pin1, peptidyl-prolyl cis/trans isomerase NIMA-interacting 1; siRNA, small interfering RNA; VEGF, vascular endothelial growth factor; MMP9, matrix metalloproteinase 9; RNAi, RNA interference; NT siRNA, non-targeting siRNA; UNT, untreated; OD, optical density.

The results of the apoptosis assay revealed an increase in apoptosis following Pin1 inhibition (Fig. 1B and C). In total, $>90 \%$ of the glioblastoma cells transfected with Pin1 siRNA entered into apoptosis, but not necrosis. By contrast, the apoptotic activity following treatment with non-targeting siRNA was significantly lower (Fig. 1C).

Inhibition of Pinl decreases VEGF and MMP9 levels. While the levels of Pin1 and VEGF were reduced in the glioblastoma cells following siRNA-mediated Pin1-knockdown, non-targeting siRNA had no marked effect on the levels of Pin1 and VEGF (Fig. 1B). The present study also demonstrated that Pin1 inhibition affected the migration and wound-healing capacities of the glioblastoma cells. The results of the wound-healing assay suggested that Pin1 may be an important molecule involved in the process of tumor cell migration, and that it may regulate MMP9 activity. Juglone treatment was also effective in blocking the migration and wound-healing capacity of the cells. Therefore, MMP9 protein levels were assessed following juglone-mediated Pin1 inhibition (Fig. 1D). The results indicated a decrease in the level of MMP9, which may explain the reduced wound-healing capacity of the cells (Fig. 1E). A decrease in Pin1 following juglone treatment was also observed, which may be the result of juglone-bound, non-functional Pin1 being targeted for degradation in the cell.

\section{Discussion}

Pin1 is a novel regulator, which functions at the center of a number of important signaling pathways that are vital for cell growth, differentiation, division and survival $(14,15)$. Pin1 is expressed at varying levels in different cell types and pathological conditions, which indicates a function that is fine-tuned to specific cell types. This makes Pin1 an 
attractive target molecule, particularly in studies concerning cancer and neurodegeneration. Furthermore, its overexpression in various human cancers makes it an effective prognostic marker (16-18). It has been established that multiple oncogenic signaling pathways involved in tumorigenesis are affected by the overexpression of Pin $1(7,17,19,20)$. Therefore, based on its differential expression patterns, Pin1 inhibition has the potential to target continuously dividing cancerous cells. Pin1-targeting agents, including juglone (8), aryl-indanyl ketones (21), D-phospho-Thr containing peptides (22), and novel and less cytotoxic Pin1 inhibitors, such as TME-001 (23), are potential candidate molecules that could be further developed during the course of drug design strategies. Notably, Pin1 has been identified to be downregulated in neurodegeneration, and has been associated with abnormal cell cycle re-entry in hippocampal neurons, eventually contributing to the neurodegenerative pathology $(24,25)$. Therefore, Pin1 may be a potential target for cell type-specific therapeutic strategies, as an adjuvant (10) or as a primary approach, that may have clinical implications in cancer and neurodegeneration.

The present study revealed that the knockdown of Pin1 altered certain tumorigenic features of glioblastoma cells through the induction of apoptosis and a decrease in cell proliferation, migration and wound-healing abilities. Following the inhibition of Pin1, a significant reduction in the growth rate of the glioblastoma cells was observed. In addition, knockdown of Pin1 significantly decreased the levels of VEGF and MMP9, which are responsible for angiogenesis and metastasis, respectively. A number of the currently available therapeutic agents used in cancer treatment depend upon anti-VEGF strategies that target either VEGF itself or its receptors (26). The present study instead targeted the transcriptional regulation of VEGF by inhibiting Pin1, since the molecular links between Pin1 and VEGF expression are relatively clear. The VEGF promoter region contains several binding sites for specific protein-1, and signal transducer and activator of transcription-3, along with HIF-1 and AP1 (27). AP1 is a transcriptional complex that is activated by Pin1 (6). Pin1 has also been identified to activate HIF-1 in an indirect manner by inactivating GSK3; a protein that phosphorylates HIF-1 for degradation (28). Pin1-mediated transcriptional activation of VEGF is known to depend upon AP1, since there are three separate binding sites for AP1 on the VEGF promoter (29).

In addition to VEGF, the regulation of MMP9 was also altered upon Pin1 inhibition in the present study. However, the possible molecular links between Pin1 and MMP9 require further elucidation. Previous studies have established that MMP9 expression is associated with glioblastoma growth and invasion (30). Recently, it has been demonstrated that the inhibition of Pin1 in colorectal carcinoma cells decreases the level and enzymatic activity of MMP9, presumably via nuclear factor- $\kappa \mathrm{B}$ signaling (13). Similarly, a reduction in MMP9 following Pin1 inhibition was observed in the present study. This decrease may have lead to a reduction in the migration and wound-healing capacity of the glioblastoma cells.

The molecular connections between Pin1 and cancer were first suggested by a study that demonstrated the overexpression of Pin1 in cancer tissues (18). Further studies confirmed the widespread overexpression of Pin1 in 60 different tumor types (3). The abundance of Pin1 in brain tumors suggests that it may be involved in the pathogenesis of glioblastoma. In addition, it has been demonstrated that the overexpression of Pin1 in malignant gliomas inhibited the apoptosis mediated by death-associated protein 6 (Daxx), an oxidative stress responsive death-associated protein, which in turn lead to tumor progression (29). Increasing evidence has indicated that Daxx has a significant role in the cellular apoptotic response induced by ultraviolet light, oxidative stress and glucose deprivation (31-33). It has been established that Pin1 binds to the phospho-Ser ${ }^{178}$-Pro motif of the Daxx protein, and that the overexpression of Pin1 results in the degradation of Daxx through the ubiquitin-proteasome pathway (3). By contrast, a previous study revealed that the inhibition of Pin1 in malignant gliomas increased oxidative stress-induced cellular responses, and therefore sensitized tumor cells to apoptosis through elevated Daxx levels (34). The apoptotic response observed in the present study following Pin1 inhibition may also be associated with an alteration in this pathway, as well as the loss of other aberrant oncogenic regulatory mechanisms induced by the overexpression of Pin1.

At present, further elucidation of the role of Pin 1 in cellular pathways in gliomas is important for the development of effective treatment strategies. Through cell type-specific targeting strategies, we believe that Pin1-targeted therapies will hold promise for the treatment of glioblastoma.

\section{Acknowledgements}

This study was supported by the Brain Research Society of Turkey/Pfizer Research Grant Award, 2009. Professor Turker Kilic was supported by the Turkish Academy of Sciences. The authors would like to thank Mr. Cemil Ozan Ceyhan for editing the manuscript.

\section{References}

1. Ohgaki H and Kleihues P: Epidemiology and etiology of gliomas. Acta Neuropathol 109: 93-108, 2005.

2. Lu KP, Hanes SD and Hunter T: A human peptidyl-prolyl isomerase essential for regulation of mitosis. Nature 380: 544-547, 1996.

3. Bao L, Kimzey A, Sauter G, Sowadski JM, Lu KP and Wang DG: Prevalent overexpression of prolyl isomerase Pin1 in human cancers. Am J Pathol 164: 1727-1737, 2004.

4. Göthel SF and Marahiel MA: Peptidyl-prolyl cis-trans isomerases, a superfamily of ubiquitous folding catalysts. Cell Mol Life Sci 55: 423-436, 1999.

5. Hunter T: Prolyl isomerases and nuclear function. Cell 92: 141-143, 1998.

6. Kim MR, Choi HS, Heo TH, Hwang SW and Kang KW: Induction of vascular endothelial growth factor by peptidyl-prolyl isomerase Pin1 in breast cancer cells. Biochem Biophys Res Commun 369: 547-553, 2008

7. Ryo A, Uemura H, Ishiguro H, Saitoh T, Yamaguchi A, Perrem K, Kubota Y, Lu KP and Aoki I: Stable suppression of tumorigenicity by Pin1-targeted RNA interference in prostate cancer. Clin Cancer Res 11: 7523-7531, 2005.

8. Hennig L, Christner C, Kipping M, Schelbert B, Rücknagel KP, Grabley S, Küllertz G and Fischer G: Selective inactivation of parvulin-like peptidyl-prolyl cis/trans isomerases by juglone. Biochemistry 37: 5953-5960, 1998.

9. Kesavapany S, Patel V, Zheng YL, Pareek TK, Bjelogrlic M Albers W, Amin N, Jaffe H, Gutkind JS, Strong MJ, Grant P and Pant HC: Inhibition of Pin 1 reduces glutamate-induced perikaryal accumulation of phosphorylated neurofilament- $\mathrm{H}$ in neurons. Mol Biol Cell 18: 3645-3655, 2007. 
10. Mathur R, Chandna S, N Kapoor P and S Dwarakanath B: Peptidy prolyl isomerase, Pin1 is a potential target for enhancing the therapeutic efficacy of etoposide. Curr Cancer Drug Targets 11: 380-392, 2011.

11. Mironova EV, Evstratova AA and Antonov SM: A fluorescence vital assay for the recognition and quantification of excitotoxic cell death by necrosis and apoptosis using confocal microscopy on neurons in culture. J Neurosci Methods 163: 1-8, 2007.

12. Ongusaha PP, Kwak JC, Zwible AJ, Macip S, Higashiyama S, Taniguchi N, Fang L and Lee SW: HB-EGF is a potent inducer of tumor growth and angiogenesis. Cancer Res 64: 5283-5290, 2004.

13. Qin L, Li M, Ren W, Zhang D, Zhang J, Zhang Y and Cheng N Silencing Pin1 suppresses the expression and bioactivity of MMP-9 through NF- $\mathrm{KB}$ in colorectal carcinoma SW480 cells. Clin Oncol Cancer Res 7: 12-17, 2010

14. Lu KP: Prolyl isomerase Pin1 as a molecular target for cancer diagnostics and therapeutics. Cancer Cell 4: 175-180, 2003.

15. Atkinson GP, Nozell SE, Harrison DK, Stonecypher MS, Chen D and Benveniste EN: The prolyl isomerase Pin1 regulates the NF-kappaB signaling pathway and interleukin-8 expression in glioblastoma. Oncogene 28: 3735-3745, 2009.

16. Ayala G, Wang D, Wulf G, Frolov A,Li R, Sowadski J, Wheeler TM, $\mathrm{Lu} \mathrm{KP}$ and Bao L: The prolyl isomerase Pin1 is a novel prognostic marker in human prostate cancer. Cancer Res 63: 6244-6251, 2003

17. Ryo A, Nakamura M, Wulf G, Liou YC and Lu KP: Pin1 regulates turnover and subcellular localization of beta-catenin by inhibiting its interaction with APC. Nat Cell Biol 3: 793-801, 2001.

18. Wulf GM, Ryo A, Wulf GG, Lee SW, Niu T, Petkova V and Lu KP Pin1 is overexpressed in breast cancer and cooperates with Ras signaling in increasing the transcriptional activity of c-Jun towards cyclin D1. EMBO J 20: 3459-3472, 2001.

19. Liou YC, Ryo A, Huang HK, Lu PJ, Bronson R, Fujimori F, Uchida T, Hunter T and Lu KP: Loss of Pin1 function in the mouse causes phenotypes resembling cyclin D1-null phenotypes. Proc Natl Acad Sci USA 99: 1335-1340, 2002.

20. Ryo A, Liou YC, Lu KP and Wulf G: Prolyl isomerase Pin1: a catalyst for oncogenesis and a potential therapeutic target in cancer. J Cell Sci 116: 773-783, 2003.

21. Daum S, Erdmann F, Fischer G, Féaux de Lacroix B, Hessamian-Alinejad A, Houben S, Frank W and Braun M: Aryl indanyl ketones: efficient inhibitors of the human peptidyl prolyl cis/trans isomerase Pin1. Angew Chem Int Ed Engl 45: 7454-7458, 2006.

22. Zhang Y, Daum S, Wildemann D, Zhou XZ, Verdecia MA, Bowman ME, Lücke C, Hunter T, Lu KP, Fischer G and Noel JP: Structural basis for high-affinity peptide inhibition of human Pin1. ACS Chem Biol 2: 320-328, 2007.
23. Mori T, Hidaka M, Lin YC, Yoshizawa I, Okabe T, Egashira S, Kojima H, Nagano T, Koketsu M, Takamiya M and Uchida T: A dual inhibitor against prolyl isomerase Pin1 and cyclophilin discovered by a novel real-time fluorescence detection method. Biochem Biophys Res Commun 406: 439-443, 2011.

24. Lee TH, Pastorino L and Lu KP: Peptidyl-prolyl cis-trans isomerase Pin1 in ageing, cancer and Alzheimer disease. Expert Rev Mol Med 13: e21, 2011

25. Atabay KD and Karabay A: Pin1 inhibition activates cyclin D and produces neurodegenerative pathology. J Neurochem 120: 430-439, 2012.

26. Sun J, Blaskovich MA, Jain RK, et al: Blocking angiogenesis and tumorigenesis with GFA-116, a synthetic molecule that inhibits binding of vascular endothelial growth factor to its receptor. Cancer Res 64: 3586-3592, 2004.

27. Xie K, Wei D, Shi Q and Huang S: Constitutive and inducible expression and regulation of vascular endothelial growth factor. Cytokine Growth Factor Rev 15: 297-324, 2004.

28. Flügel D, Görlach A, Michiels C and Kietzmann T: Glycogen synthase kinase 3 phosphorylates hypoxia-inducible factor 1alpha and mediates its destabilization in a VHL-independent manner. Mol Cell Biol 27: 3253-3265, 2007.

29. Chabannes E, Fauconnet S, Bernardini S, Wallerand H, Adessi $\mathrm{G}$ and Bittard $\mathrm{H}$ : Protein kinase $\mathrm{C}$ signalling pathway is involved in the regulation of vascular endothelial growth factor expression in human bladder transitional carcinoma cells. Cell Signal 13: 585-591, 2001.

30. Choe G, Park JK, Jouben-Steele L, Kremen TJ, Liau LM, Vinters HV, Cloughesy TF and Mischel PS: Active matrix metalloproteinase 9 expression is associated with primary glioblastoma subtype. Clin Cancer Res 8: 2894-2901, 2002.

31. Zhong S, Salomoni P, Ronchetti S, Guo A, Ruggero D and Pandolfi PP: Promyelocytic leukemia protein (PML) and Daxx participate in a novel nuclear pathway for apoptosis. J Exp Med 191: 631-640, 2000

32. Perlman R, Schiemann W, Brooks MW, Lodish HF and Weinberg RA: TGF-beta-induced apoptosis is mediated by the adapter protein Daxx that facilitates JNK activation. Nat Cell Biol 3: 708-714, 2001

33. Salomoni P and Khelifi A: Daxx: death or survival protein? Trends Cell Biol 16: 97-104, 2006.

34. Ryo A, Hirai A, Nishi M, Liou YC, Perrem K, Lin SC, Hirano H, Lee SW and Aoki I: A suppressive role of the prolyl isomerase Pin1 in cellular apoptosis mediated by the death-associated protein Daxx. J Biol Chem 282: 36671-36681, 2007. 